\title{
VARIAÇÃO ESPAÇO-TEMPORAL DA OCORRÊNCIA DO FOGO NOS BIOMAS BRASILEIROS COM BASE NA ANÁLISE DE PRODUTOS DE SENSORIAMENTO REMOTO
}

\author{
Daniel Borini ALVES ${ }^{1}$ \\ Swanni T. ALVARADO²
}

\begin{abstract}
RESUMO
O estudo das relações entre o fogo e a dinâmica das paisagens brasileiras resulta fundamental para uma melhor compreensão dos processos ecológicos e dos impactos humanos incidentes sobre a dinâmica da paisagem. O presente artigo busca explorar a dinâmica de variação espaço-temporal de ocorrência de queimadas nos biomas brasileiros nos últimos 18 anos (2001-2018), com base no produto MCD64A1 voo6 de área queimada e a base de dados de uso e cobertura da terra do projeto Mapbiomas. O Cerrado apresentou 62,2\% do total queimado registrado no período analisado, seguido do bioma Amazônia, com 23,5\%. Pampa, Mata Atlântica e Amazônia apresentaram uma incidência de queimadas superior a $\approx 50 \%$ em áreas com usos da terra agropastoris, enquanto nos biomas Cerrado, Caatinga e Pantanal as queimadas ocorreram principalmente sobre áreas de formação vegetal natural. Comprova-se o potencial de combinação de uso de produtos derivados de sensoriamento remoto para análise dos padrões de áreas queimadas, gerando-se relevantes informações a respeito das influências antrópicas na alteração dos regimes de fogo.
\end{abstract}

Palavras-chave: Áreas queimadas. Biomas brasileiros. Sensoriamento remoto. MCD64A1. Mapbiomas

\section{SPATIO-TEMPORAL VARIATION OF FIRE OCCURRENCE IN BRAZILIAN BIOMES BASED ON THE ANALYSIS OF REMOTE SENSING PRODUCTS}

\begin{abstract}
The study of the relationship between fire and the dynamics of Brazilian landscapes is a major issue for a better understanding of ecological processes and the human impacts on landscape dynamics. We explore the dynamics of spatial-temporal variation in fire occurrence in Brazilian biomes during the last 18 years (2001-2018), based on the burned area product MCD64A1 voo6 and

\footnotetext{
${ }^{1}$ Doutor em Geografia pela Universidad de Zaragoza (UNIZAR/Espanha). Pós-doutorando no Departamento de Biodiversidade da Universidade Estadual Paulista "Júlio de Mesquita Filho" (UNESP/Rio Claro, SP). E-mail: Daniel.borini@unesp.br

2 Doutora em Ciências da Vida pela Université d'Avignon et des Pays de Vaucluse (UAPV/França). Pós-doutoranda no Programa de Pós-graduação em Agricultura e Ambiente, Universidade Estadual do Maranhão (UEMA/Balsas, MA); e docente no Programa de Pós-graduação em Geografia, Natureza e Dinâmica do Espaço (UEMA/São Luís, MA). E-mail: swanni_ta@yahoo.es
} 
the database of land use and land cover of Mapbiomas project. The Cerrado recorded $62.2 \%$ of the total burned in the analyzed period, followed by the Amazon biome, with 23.5\%. Pampa, Mata Atlântica and Amazônia had a fire occurrence above $\approx 50 \%$ in areas with agropastoral land uses, while in the Cerrado, Caatinga and Pantanal biomes the fires occurred mainly on areas of natural vegetation. We confirmed the potential for combining the use of products derived from remote sensing for the analysis of the patterns of burned areas, generating relevant information regarding the anthropic influences in the alteration of fire regimes.

Keywords: Burned areas. Brazilian biomes. Remote sensing. MCD64A1. Mapbiomas.

\section{VARIACIÓN ESPACIO-TEMPORAL DE LA OCURRENCIA DE INCENDIOS EN BIOMAS BRASILEÑOS BASADA EN EL ANÁLISIS DE PRODUCTOS DE TELEDETECIÓN}

\section{RESUMEN}

El estudio de la relación entre el fuego y la dinámica de los paisajes brasileños es fundamental para una mejor comprensión de los procesos ecológicos y los impactos humanos en la dinámica del paisaje. Este artículo busca explorar la dinámica de la variación espacio-temporal en la ocurrencia de incendios en los biomas brasileños en los últimos 18 años (2001-2018), con base en el producto MCD64A1 voo6 de área quemada y la base de datos de usos y cobertura de la tierra del proyecto Mapbiomas. El Cerrado presentó el 62.2\% del total quemado registrado en el período analizado, seguido por el bioma amazónico, con el 23.5\%. Pampa, Mata Atlántica y Amazonia tuvieron una incidencia de incendios por encima del $50 \%$ en áreas con uso de tierras agropastorales, mientras que en los biomas de Cerrado, Caatinga y Pantanal los incendios ocurrieron principalmente en áreas de vegetación natural. Se demuestra el potencial para combinar el uso de productos derivados de la teledetección para el análisis de los patrones de áreas quemadas, generando información relevante sobre las influencias antrópicas en la alteración de los regímenes de incendios.

Palabras clave: Áreas quemadas. Biomas brasileños. Teledetección. MCD64A1. Mapbiomas.

\section{INTRODUÇÃ̃o}

Na busca de uma ampla compreensão da dinâmica dos processos naturais e das influências antrópicas que incidem sobre a superfície terrestre, entender os efeitos e repercussões do fogo na formação e transformação das paisagens se torna um importante desafio científico no âmbito da Geografia. Muitas vezes desconsiderado ou infra-estimado nos modelos tradicionais de análise da 
integração dos elementos na paisagem, o fogo é um dos elementos chave na dinâmica dos ecossistemas terrestres (BOND; WOODWARD; MIDGLEY, 2005; BOWMAN et al., 2009; PAUSAS; KEELEY, 2009; HE; LAMONT; PAUSAS, 2019).

Registros paleoclimáticos e paleobotânicos comprovam a presença de regimes naturais de queima na evolução dos ecossistemas terrestre muito antes da emergência dos humanos, estando sua origem vinculada ao surgimento das plantas, há $\approx 420$ milhões de anos atrás (SCOTT; GLASSPOOL, 2006). O surgimento e expansão do homem o consolidou como a principal fonte de ignições de queimadas na Terra, superando as ignições naturais originadas por raios ou outra fonte de calor, e resultando na alteração significativa dos regimes naturais de fogo, principalmente pelo aumento da frequência de queimadas (GOLDAMMER, 1993; PAUSAS; KEELEY, 2009). Para além do aumento das ignições diretas, as transformações da paisagem promovidas pelo homem modificam as suas condições de susceptibilidade ao fogo, materializadas principalmente pela construção de novas redes viárias e pelo avanço das fronteiras agropecuárias, com remoção da vegetação natural, introdução de espécies invasoras e uso do fogo como ferramenta agropastoril (NEPSTAD et al., 2001).

Ainda que o fogo seja um importante elemento natural da dinâmica dos processos ecológicos (PIVELLO, 2011), alterações significativas nos regimes naturais de queima, que refletem em níveis elevados de recorrência de fogo e favorecem a ocorrência de incêndios em períodos críticos de seca (ARAGÃO et al., 2008), podem gerar desequilíbrios em distintos componentes do ecossistema. Podem resultar, por exemplo, na exclusão de determinadas espécies mais sensitivas ao fogo (MOREIRA, 2000; HOFFMANN; MOREIRA, 2002), no aumento das taxas de erosão e na alteração da composição química e física dos solos (CERTINI, 2005; PÉREZ-CABELLO et al., 2009; ALCAÑIZ et al., 2016) e em níveis elevados de emissão de gases de efeito estufa (LEVINE et al., 1995; VAN DER WERF et al., 2010). Tudo isso sem contar nos graves prejuízos sociais e econômicos aos que podem estar associados, como se observou em episódios recentes de grandes e destrutivos incêndios em países como Portugal (junho e outubro de 2017, 109 mortos), Grécia (julho de 2018, com 100 mortos), Estados 
Unidos (novembro de 2008, com 88 mortos) e Austrália (setembro de 2019 a fevereiro de 2020, com 32 mortos), sendo os prejuízos econômicos deste último contabilizados em aproximadamente 5 bilhões de dólares (CRED, 2018, 2019, 2020).

Porém, os impactos das alterações dos regimes de fogo alcançam distintos patamares de acordo com as características de determinado ecossistema afetado, o que em termos ecológicos guarda muita relação com a forma como determinada paisagem evoluiu historicamente com o fogo. Neste contexto, Hardesty, Myers e Fulks (2005) categorizam os ecossistemas em três grupos: fire sensitive, fire independent e fire dependent/influenced. O primeiro grupo inclui áreas que são sensíveis a ocorrência de incêndios, tais como as florestas tropicais da bacia Amazônica, onde grande parte da sua biota não possui mecanismos de adaptação para responder ou se recuperar rapidamente do fogo. Os ecossistemas independentes do fogo são aqueles em que ele não ocorre, ou ocorre raramente, em função da indisponibilidade de material combustível, como por exemplo o deserto da Namíbia ou a tundra da costa Antártica. Por fim, o terceiro grupo se refere aos ecossistemas considerados dependentes/influenciados pelo fogo, que apresentam fitofisionomias que respondem a esta perturbação com alta capacidade de resiliência e recuperação, tais como as savanas tropicais da América do Sul (HARDESTY; MYERS; FULKS, 2005).

Nesta última categoria, alterações de regimes de fogo relacionadas a políticas concentradas na exclusão do fogo podem gerar impactos significativos, afetando negativamente espécies típicas de áreas abertas com o adensamento de espécies lenhosas (“woody encroachment”) (ARCHER et al., 2017; STEVENS et al., 2017), e favorecendo a ocorrência de grandes incêndios pelo maior acúmulo de material combustível das áreas (FIDELIS et al., 2018; SCHMIDT et al., 2018).

$\mathrm{Na}$ vasta extensão do território brasileiro, é possível identificar ecossistemas que apresentam características diferentes em relação as respostas ao fogo. Biomas tais como o Cerrado, Pampa e Pantanal seriam considerados como fire dependent/influenced devido as características da sua vegetação dominada principalmente por gramíneas e ao seu histórico 
evolutivo relacionado com a presença do fogo (SIMON et al., 2009; PIVELLO, 2011); por outro lado, biomas com vegetação dominantemente florestal, como a Mata Atlântica e a Amazônia seriam considerados como fire sensitive; e finalmente a Caatinga, ainda que com poucos estudos que remarcam a sua relação histórica com o fogo, poderia ser considerado como fire independent (PIVELLO, 2011). No Brasil, as áreas de transição entre os biomas Cerrado e Amazônia são consideradas como as mais frequentemente afetadas pelo fogo nas últimas décadas (SCHROEDER et al., 2009; SILVA CARDOZO et al., 2011), onde o aumento de registros de áreas queimadas está fortemente relacionado com alterações de uso e cobertura da terra, principalmente no que se refere a áreas de expansão e consolidação das fronteiras agropecuárias (EVA; LAMBIN, 2000; OMETTO; SOUZA-NETO; TEJADA, 2016).

Nesta perspectiva, revela-se a importância de uma adequada caracterização dos padrões espaço-temporais de incidência de fogo existentes sobre distintos ecossistemas, para uma melhor compreensão dos processos ecológicos e dos impactos humanos incidentes sobre a dinâmica da paisagem. Para analisar os regimes de queimadas em múltiplas escalas espaciais (local/regional/continental), o uso de produtos derivados de sensoriamento remoto se consolida como uma importante fonte de dados, ao disporem de informações de vastas áreas com um recobrimento multitemporal e multiespectral (GIGLIO et al., 2010). Há neste sentido uma vasta literatura científica concentrada na reconstrução e análise espacial de históricos de fogo utilizando produtos de sensoriamento remoto, tais como os estudos realizados em áreas de savana de África do Sul e Botsuana (HUDAK; BROCKETT, 2004) ou Madagascar (JACQUIN; GOULARD, 2013; ALVARADO; SILVA; ARCHIBALD, 2018), em ambientes campestres áridos da Austrália (GREENVILLE et al., 2009), vegetação mediterrânea de Israel (LEVIN; HEIMOWITZ, 2012) e Espanha (RÖDER et al., 2005), florestas tropicais da Amazônia (ALENCAR et al., 2011; MORTON et al., 2011), Cerrado (DALDEGAN et al., 2014; LEMES et al., 2014; ALVARADO et al., 2017; BATISTA et al., 2018), savana amazônica (ALVES; PÉREZ-CABELLO, 2017), e fisionomias de 
transição entre o Cerrado e a Caatinga (ARGIBAY; SPARACINO; ESPINDOLA, 2020).

Para além dessas iniciativas de reconstrução de históricos regionais de fogo, observa-se nos últimos anos um panorama de notável evolução dos produtos globais de detecção automática de áreas queimadas derivados de informações de satélite (MOUILLOT et al., 2014). Entre estes produtos, destacam-se aqueles derivados da série dos sensores Moderate Resolution Imaging Spectroradiometer (MODIS), e em especial o produto MCD64A1 (GIGLIO et al., 2018), que apresenta uma boa performance em relação a outros produtos de moderada resolução espacial disponíveis na literatura (TSELA et al., 2014; PADILLA et al., 2015; HUMBER et al., 2018). Informações de áreas queimadas deste produto são utilizadas, por exemplo, como importantes inputs na contabilização de emissões de gases de efeito estufa da Global Fire Emissions Database (VAN DER WERF et al., 2017).

Mas os avanços de produtos de sensoriamento remoto não se restringem a aqueles relacionados a detecção de áreas queimadas, sendo outro exemplo significativo a derivação de produtos que permitem captar variações espaçotemporais de uso e cobertura da terra. No Brasil, um importante produto se destaca entre os atualmente disponíveis, derivado de uma iniciativa multiinstitucional para mapear anualmente o uso de cobertura da terra dos biomas brasileiros de 1985 até os anos atuais com imagens de média/alta resolução espacial (baseado nas coleções de imagens Landsat), conhecido como Mapbiomas (MAPBIOMAS, 2019). A combinação de produtos de áreas queimadas com os de dinâmica de uso e cobertura da terra permite dispor de informações importantes para a caracterização dos regimes atuais de queima, sendo um relevante aliado para a compreensão das influências antrópicas nas suas alterações.

Neste contexto, o presente artigo busca explorar a dinâmica de variação espaço-temporal de ocorrência de queimadas nos biomas brasileiros nos últimos 18 anos (2001-2018), com base na sistematização e análise de produtos de sensoriamento remoto. Quais são os padrões de incidência do fogo nos biomas brasileiros em relação aos tipos de superfícies queimadas? Esta é a questão específica que se propõe responder 
ao longo do texto, gerando-se relevantes informações a respeito das relações entre o fogo e a dinâmica das paisagens brasileiras.

\section{MATERIAIS E MÉTODOS}

\section{Descrição dos produtos de sensoriamento remoto utilizados}

Conta-se com dois principais datasets de entrada para dar resposta aos objetivos implementados: os dados de áreas queimadas do produto MCD64A1, versão 6; e as informações de uso e cobertura da terra derivadas do MapBiomas Coleção 4.1. A escala temporal de análise está adequada a disponibilidade da série anual completa coincidente dos dois produtos utilizados, ou seja, com os dados de 2001 a 2018 (período de 18 anos).

O produto MCD64A1 voo6, denominado Direct Broadcast Monthly Burned Area Product (GIGLIO et al., 2016, 2018), é o mais recente produto de detecção de áreas queimadas da série MODIS, incluído na sua Coleção VI (disponível em: $<$ https://modis.gsfc.nasa.gov/data/dataprod/>). Com uma resolução espacial de 500 metros, o produto é disponibilizado mensalmente (desde o mês de novembro de 2000 até os dias atuais), sendo proporcionado para cada pixel valores de o a 366 : onde o corresponde a um pixel não queimado; enquanto que os valores de 1 a 366 indicam a data juliana de detecção de queima em determinado pixel. Este produto é gerado a partir de um algoritmo automatizado que detecta rápidos padrões de alterações na série temporal de refletividade de superfície detectada pelos sensores MODIS. Se diferencia do anterior produto da coleção, o MCD45A1 (ROY; LEWIS; JUSTICE, 2002), ao integrar no seu algoritmo o uso de informações de focos ativos de fogo para gerar funções de densidade de probabilidade adequadas regionalmente a detecção da persistência das mudanças observadas na série de refletividade.

Por outro lado, o Mapbiomas (MAPBIOMAS, 2019) é uma iniciativa que envolve universidades, ONGs e empresas de tecnologia com o propósito de mapear anualmente a cobertura e uso da terra do Brasil e monitorar as mudanças do território (disponível em: <https://mapbiomas.org/>). A Coleção 4.1, otimizada em relação as suas anteriores versões, oferece produtos de resolução espacial de 30 metros, com mapas anuais de uso de cobertura da terra desde 1985 a 2018 para todos os biomas brasileiros, além de produtos derivados 
da análise das mudanças espaciais entre categorias específicas do mapeamento. Baseia-se na aplicação de algoritmos automáticos sobre a série imagens dos satélites Landsat, de qual se derivam mosaicos de imagens anuais e se aplicam classificadores baseados na lógica de aprendizagem de máquina (random forest classifier). Passam-se ainda por rotinas de pós-processamento (filtros espaciais e de integração temporal) para reduzir inconsistências das classificações geradas.

\section{Procedimentos e processos de análise}

Os procedimentos metodológicos estão divididos em duas principais etapas: i) obtenção e processamento dos produtos de sensoriamento remoto; ii) estratificação anual e análise descritiva dos padrões de áreas queimadas, por biomas e segundo o uso e cobertura da terra.

\section{Obtenção e preparação dos produtos de sensoriamento remoto}

Como alternativa para superar as limitações associadas com a obtenção e processamento de séries históricas longas derivadas de sensores remotos em escala nacional, atualmente se conta com o surgimento de plataformas online de processamento de informações espaciais, com destaque para a Google Earth Engine (GEE) (GORELICK et al., 2017). Nesta plataforma, as imagens estão disponíveis em servidores online que permitem o seu acesso e processamento mediante codificação, em linguagem de programação JAVA, o que reduz consideravelmente o tempo e a exigência de hardware por parte do usuário. Ambos os produtos de sensoriamento remoto utilizados estão disponíveis na plataforma GEE, na qual foram obtidos gratuitamente.

Na preparação do produto de áreas queimadas MCD64A1 voo6, foi elaborado um script GEE para gerar uma imagem acumulada anual a nível nacional, subdividindo-se por biomas com base no arquivo vetorial 1:250.000 disponibilizado pelo Instituto Brasileiro de Geografia e Estatística (IBGE). O código implementado acessa e mosaica todas as imagens mensais do produto para determinada área e período indicados, gerando-se um único arquivo raster acumulado que identifica cada pixel como queimado/não queimado (pixels com 
valores de 1 e o respetivamente), com base nos valores do layer "Burn Date" do produto.

Já o acesso aos arquivos de uso e cobertura da terra pelo GEE se fez através de uma ferramenta (<https://github.com/mapbiomas-brazil/user-toolkit>) compartilhada pela equipe do Mapbiomas. Obteve-se os rasters anuais de tipos de uso e cobertura da terra para cada bioma estudado.

\section{Estratificação anual e análise descritiva dos padrões de áreas queimadas}

Primeiramente foi realizado um mapa de frequência de queimadas a nível nacional, baseado nos dados anuais do produto MCD64A1 voo6, mantendo a sua resolução espacial original (500m). Estas informações foram sobrepostas mediante álgebra de mapas (operação de soma), com o objetivo de calcular a frequência do fogo por pixel no intervalo temporal selecionado.

Posteriormente, com base nos rasters anuais de área queimada e uso e cobertura da terra gerados na etapa anterior, passou-se a estratificar as informações combinadas dos dados. Considerando as limitações inerentes a combinação de produtos de diferentes resoluções espaciais (Mapbiomas - 30m vs. MCD64A1 voo6 - 500m), a contabilização anual se restringe a uma análise estatística descritiva que verifica que tipos de uso e cobertura predominam nos pixels de menor resolução espacial registrados como queimados.

Para garantir melhor eficiência de processamento, a contabilização das informações de uso e cobertura da terra foram extraídas apenas para áreas identificadas como queimadas em determinado ano. Para isso, foi implementado um script em ambiente Python 2.7.12, que poligoniza os dados de áreas queimadas e extrai a frequência de classes do mapa de uso e cobertura da terra por ano e bioma. Para fins de otimizar a interpretação, algumas classes de uso e cobertura da terra foram combinadas: "Cultura anual perene" e "Cultura anual semi-perene" foram integrados em uma única classe - "Agricultura"; a categoria "Mangue" foi integrada na classe de "Formação florestal"; "Apicum" foi integrada na classe "outra formação natural não florestal”; "Afloramento rochoso", "Praias e dunas”, "Mineração" e "Outras áreas não vegetadas" foram integrados na categoria "Outros". 
Classes de rios e reservatórios de água foram mascaradas (associadas a "no data”) para evitar a sua incorreta contabilização quando existentes dentro de um pixel de menor resolução espacial classificado como queimado.

Foi calculada a área total queimada acumulada durante o período $2001 \mathrm{e}$ 2018 para cada bioma, assim como a porcentagem de área queimada por cada classe de uso e cobertura, ponderando esta porcentagem com respeito à área total de cada bioma. A abordagem gráfica se realizou utilizando o software $\mathrm{R}$ 3.6.2 (RCT, 2019), mais precisamente através do package "ggplot2", enquanto que a álgebra de mapas e geração de produtos cartográficos se obteve junto ao Sistema de Informações Geográficas (SIG) Arcmap 10.5.

\section{RESULTADOS}

Nos 18 anos de análise (2001-2018), o produto MCD64A1 voo6 detectou como queimado um total de 3,42 milhões de $\mathrm{km}^{2}$ no território nacional (Figura 1a). Em termos absolutos, o bioma Cerrado é onde mais áreas queimadas foram identificadas, com $62,2 \%$ do total registrado no período, seguido dos registros no bioma Amazônia (23,5\% do total). Destaque também para os registros do Pantanal (com $195.999 \mathrm{~km}^{2}$ queimados, 5,7\% do total), em que, mesmo sendo o menor entre os biomas brasileiros, registrou totais de área queimada superiores aos registrados na Caatinga $(2,25 \%)$ e no Pampa $(0,12)$, e similares ao total registrado na Mata Atlântica $\left(213.667 \mathrm{~km}^{2}\right)$. 
Figura 1 - Área queimada total por bioma brasileiro no período de 2001-2018, em termos absolutos, registradas no produto MCD64A1 voo6 (a), e suas respectivas correspondências de uso e cobertura da terra, em termos relativos, observados na Coleção 4.1 do Mapbiomas (b).

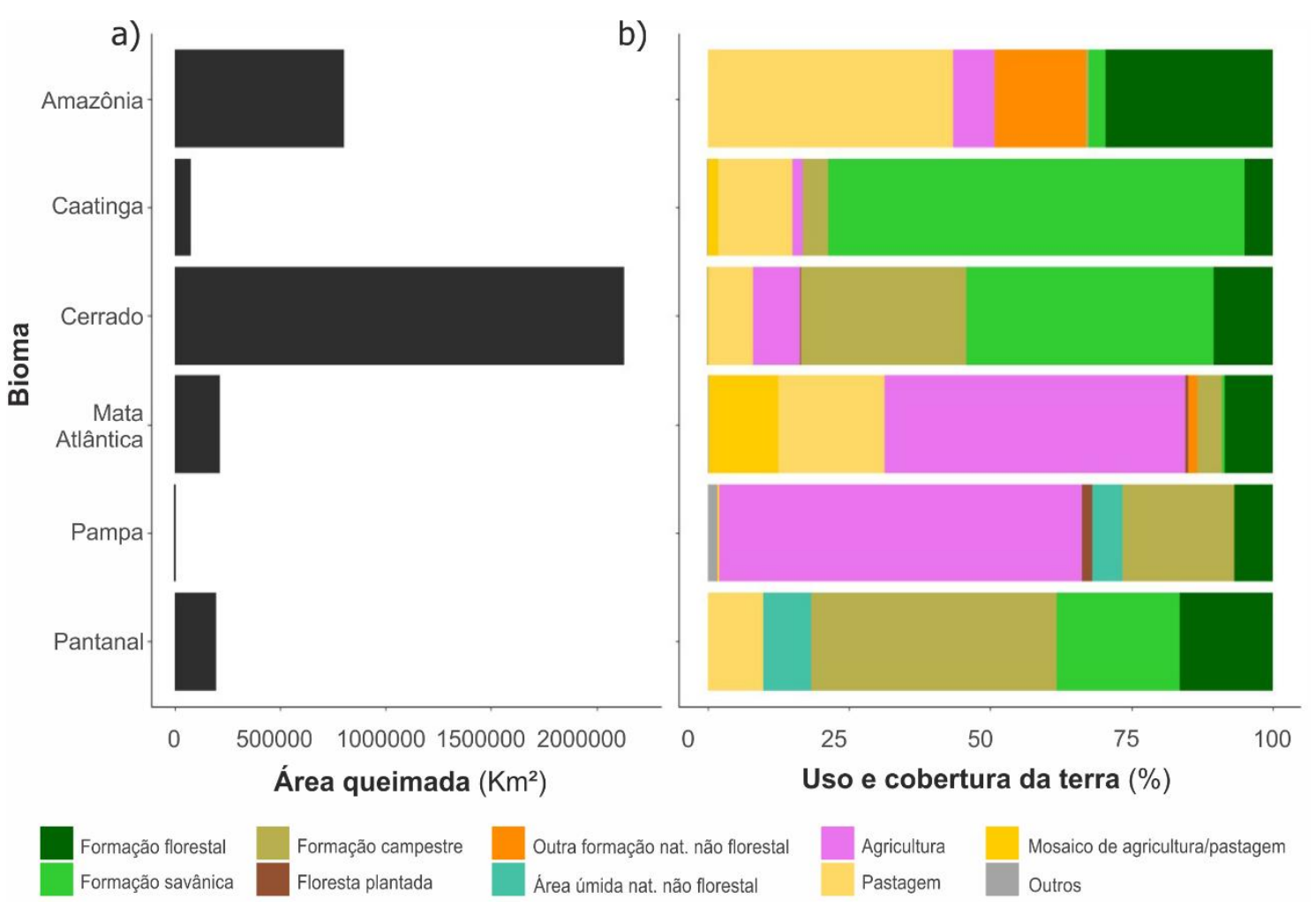

Em relação aos tipos de superfície sobre as quais se registram áreas queimadas (Figura 1b), evidencia-se nos biomas Pampa, Mata Atlântica e Amazônia a forte influência antrópica nos regimes de queima ali incidentes. Nestas regiões, os usos da terra agropastoris correspondem a altas proporções das áreas com registros de queimadas, com destaque para o Pampa (64,3\% do total). Em contrapartida, nos biomas Cerrado, Caatinga e Pantanal as queimadas sobre áreas de formação vegetal natural predominam.

A estratificação anual dos dados (Figura 2) reforça o padrão observado na análise compilada, sendo que as proporções de classes de uso e cobertura afetadas são praticamente reproduzidas anualmente. Observa-se que as categorias predominantes nas áreas queimadas (Figura 2a) não guardam necessariamente relação com a distribuição de abundância das categorias no bioma como um todo (Figura 2b). O exemplo concreto está na Amazônia, onde apesar das pastagens ocuparem aproximadamente 12,7\% do total do bioma, 
corresponde sempre a altas proporções dos estratos anuais queimados (43,5\% do total absoluto).

Figura 2 - Estratificação anual de áreas queimadas e tipos de usos e cobertura correspondente por bioma brasileiro, em porcentagens relativas ao tamanho de cada bioma (a). À direita de cada gráfico anual, apresenta-se a distribuição de categorias de uso e cobertura da terra de cada bioma, no ano de 2018, considerando a extensão total de suas áreas (b).

a)
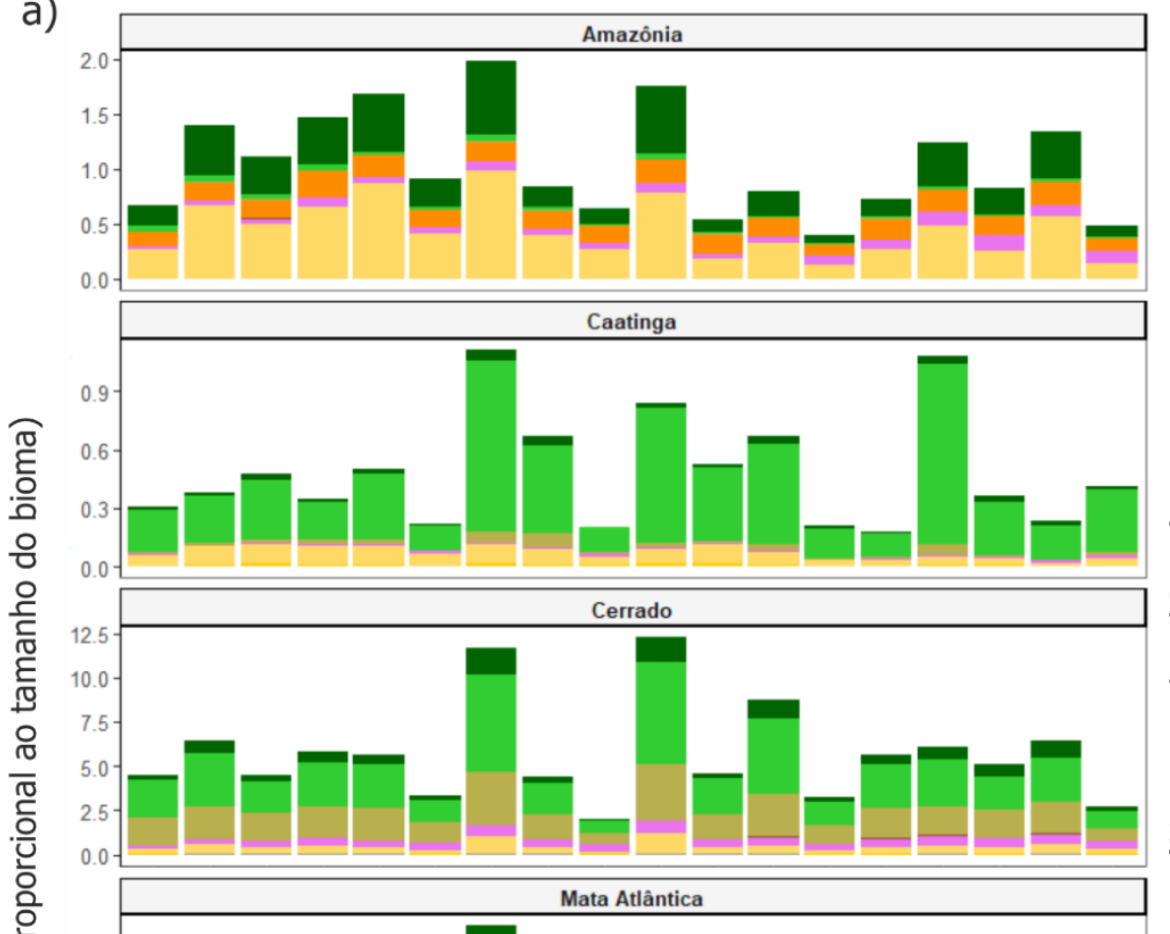

은

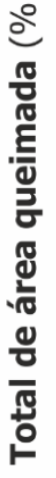

$0.0-$
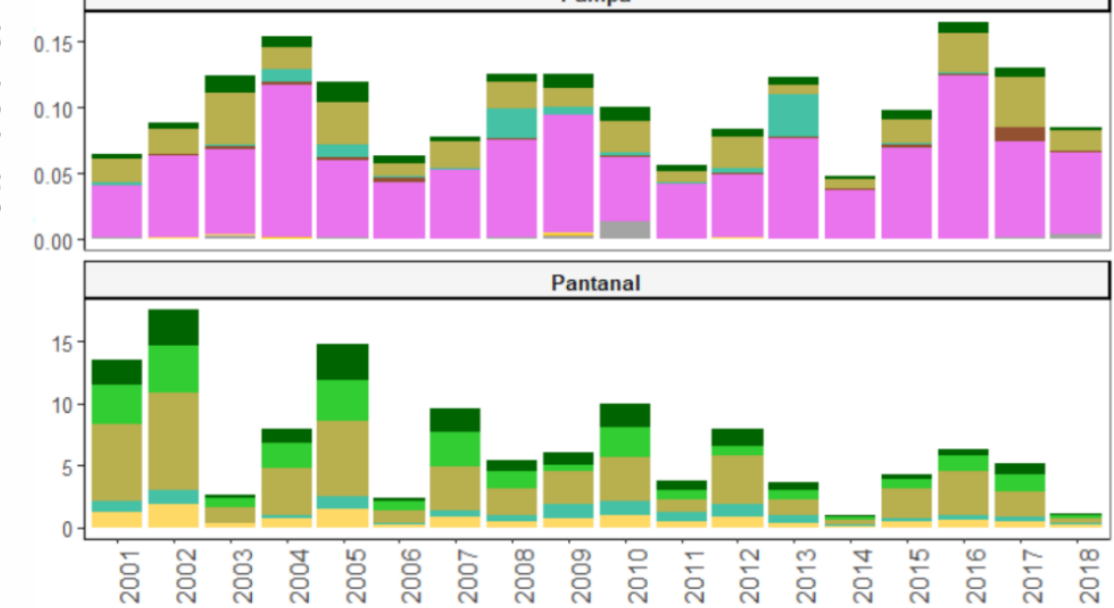

b)
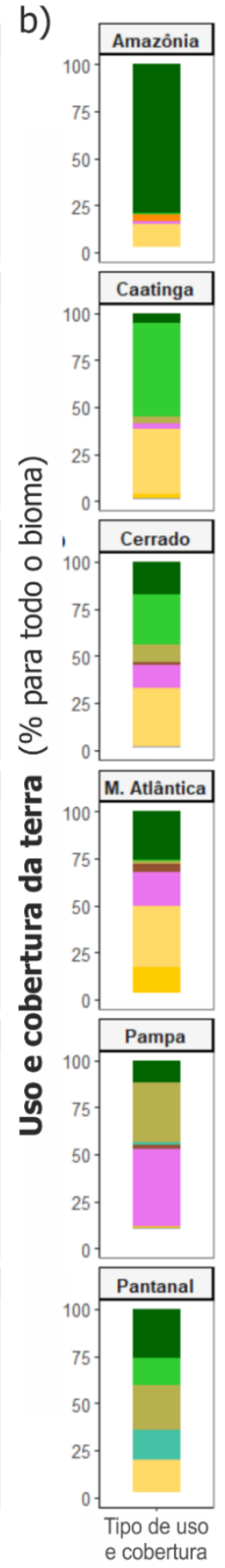

Formação florestal

Formação campestre

Outra formação nat. não florestal

Agricultura Pastagem

Mosaico agricultura/pastagem 
Na variação anual de áreas queimadas, observa-se que alguns picos são comuns em distintos biomas, tais como o ano de 2007 e 2010, bem notáveis na Amazônia, Cerrado, Caatinga e Pantanal. Outra observação sobre esses dois picos é que, em certos casos, são precedidos de um ano em que os subtotais de áreas queimadas são bem inferiores à média geral, vide-se Cerrado e Caatinga. Os padrões de variação anual de queimadas dos biomas Pampa e Mata Atlântica aparentam ter uma dinâmica mais particular em relação aos picos e descensos observados nos outros biomas, sendo em ambos predominantes as queimadas associadas a agricultura.

Em termos de incidência espacial (Figura 3), é possível observar padrões heterogêneos de queimas nos distintos biomas brasileiros e suas transições. Em áreas entre a Amazônia e o Cerrado (exemplo de fragmento na Figura 3b), observam-se mosaicos frequentemente afetados pelo fogo, com certas áreas em que o fogo foi registrado mais de 10 vezes nos 18 anos analisados. Esta área exemplificada está situada entre os estados do Tocantins e Mato Grosso, onde se registram mosaicos maiores e mais contínuos de áreas queimadas a leste, que integram áreas da ilha do Bananal; já a oeste o padrão mais frequente e fragmentado de queimadas está relacionado a áreas com extensa cobertura de pastagens, vinculados a um histórico recente de avanço das fronteiras agropecuárias na região.

Este contraste entre áreas com mosaicos de áreas queimadas mais contínuos e fragmentados também é observado em certas áreas do bioma Amazônia (Figura 3d). Áreas de enclave de Cerrado (savanas amazônicas) como o exemplo do situado no Parque Nacional dos Campos Amazônicos (a nordeste do fragmento exemplificado), apresentam mosaicos com queimas de maior extensão, contabilizados como "Outra formação natural não florestal" pelo Mapbiomas. Por outra parte, nas demais áreas se observam padrões mais fragmentados de áreas queimadas, com mosaicos mais regulares/retilíneos, associados principalmente a atividade e fogo em pastagens situadas em proximidades de vias de acesso.

As transições entre o Cerrado e a Caatinga também apresentaram relevante influência do fogo no período analisado, tal como observado na área selecionada situada entre o sul do Piaú e o noroeste da Bahia (Figura 3c). Áreas 
classificadas como "Formações savânicas" predominam entre as zonas afetadas, em grande parte queimadas entre 2 e 4 vezes na série temporal analisada.

Figura 3 - Áreas detectadas como queimadas pelo produto MCD64A1 voo6 entre os anos de 2001-2018, nos biomas brasileiros (a). Os quadros de zoom (bf) permitem observar com maior detalhe os padrões espaciais de incidência do fogo em determinadas áreas selecionadas.
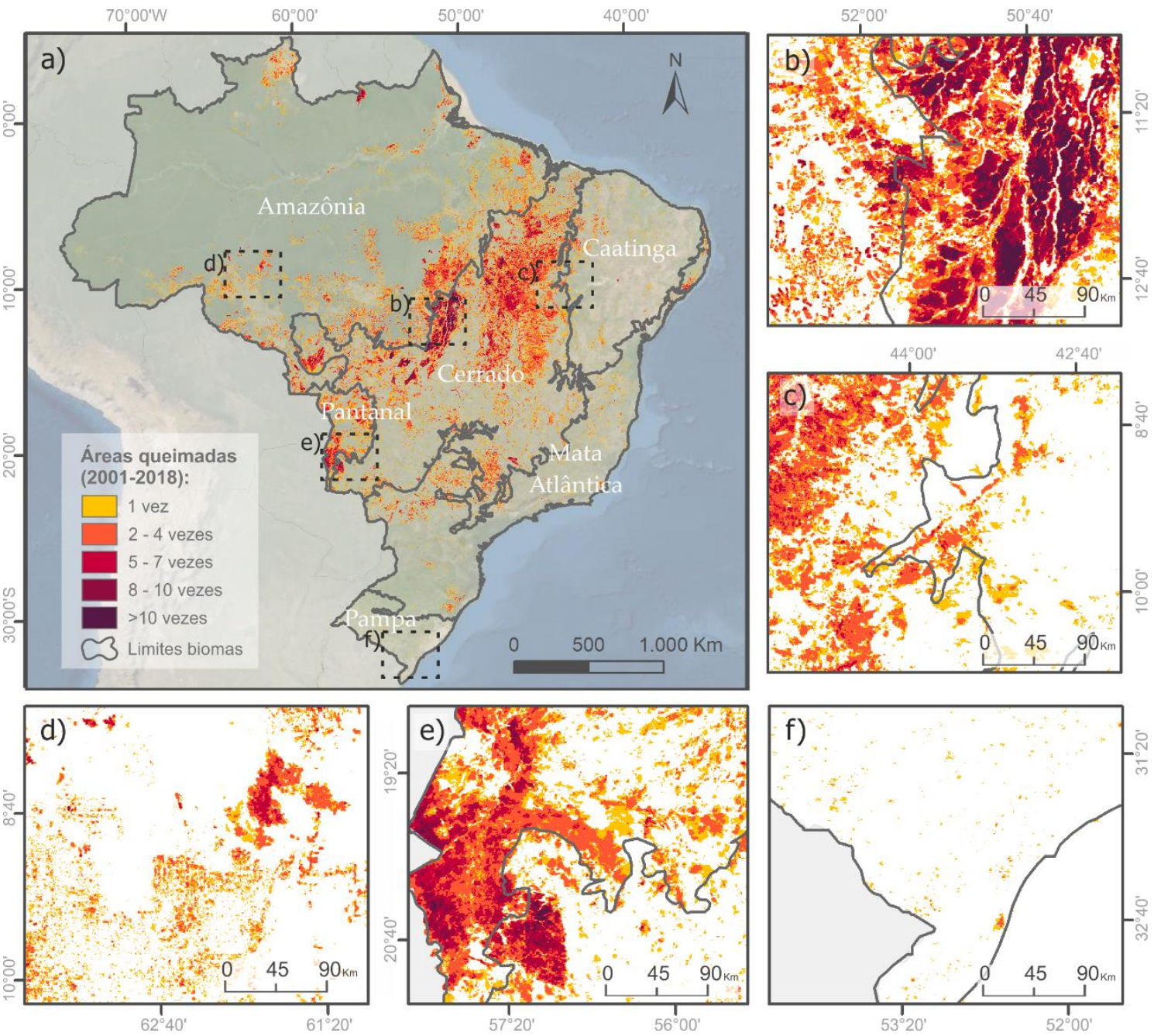

Mosaicos extensos de áreas queimadas são bem aparentes a escala nacional no bioma pantanal e zonas de transição com o Cerrado. A região selecionada (Figura 3e) se situa na porção oeste do Mato Grosso, delimitado a oeste pela fronteira com o Paraguai e Bolívia (rio Paraguai), apresentando áreas afetadas por extensas e frequentes queimadas (>8 vezes) entre 2001 e 2018.

Um último destaque pode ser apresentado em áreas do bioma Pampa (Figura 3f), onde no comparativo com as demais áreas selecionadas observa-se 
uma atividade de fogo bem menor. Os maiores mosaicos de áreas queimadas deste bioma se observam a sudeste da área selecionada, mais precisamente em áreas da Estação Ecológica do Taim, que apresenta certas formações naturais úmidas não florestais. Um padrão de queimadas menores e mais dispersas é observado em outras áreas do bioma, associado principalmente a áreas de cultivos agrícolas e pastagens.

\section{DISCUSSÃO}

Ao considerarmos a divisão regional de biomas brasileiros para estratificar a análise dos padrões de queimas no Brasil, ficam evidentes alguns aspectos relacionados aos tipos de uso e cobertura da terra sobre as quais ocorrem queimadas nos distintos ecossistemas brasileiros. Nos biomas Cerrado, Caatinga e Pantanal, os subtotais de áreas queimadas se concentram bastante em áreas de formações naturais (especialmente nas formações savânicas e campestres), enquanto que nos biomas Amazônia, Mata Atlântica e Pampa fica ressaltado a presença do fogo relacionada a atividades agrícolas e pastagens.

No caso específico da Amazônia, observa-se uma forte influência de áreas historicamente convertidas de florestas tropicais a pastagens nos estratos anuais de áreas queimadas, concentrados principalmente nas áreas de transição com o Cerrado. Estas relações sobre a presença do fogo tanto na conversão, como no manejo de pastagens posteriores a conversão, é já bastante explorado na literatura científica (NEPSTAD et al., 2001; ADENEY; CHRISTENSEN; PIMM, 2009; CARDOZO et al., 2014). Mesmo em áreas que o fogo não necessariamente está associado a conversão para áreas de pastagem ou agrícola, os efeitos das queimas também podem refletir na degradação das florestas, como demonstram resultados de experimentos que comparam áreas não queimadas com áreas queimadas com diferentes frequências (BALCH et al., 2013; BRANDO et al., 2014). Em um estudo realizado para as extensões dos estados do Pará, Mato Grosso e Rondônia, também se denota a forte relação pastagens com a presença do fogo, ressaltando que grande parte das áreas de florestas queimadas são associadas a queimas iniciadas em áreas de pastagens (CANO-CRESPO et al., 2015). 
Já os ecossistemas que possuem uma relação evolutiva com o fogo, tais como o Cerrado e o Pantanal, é preciso avançar em uma compreensão mais ampla da influência desse elemento para entender a complexidade da sua incidência espacial. Por sua dimensão e pela relevância do fogo na dinâmica dos ecossistemas savânicos, o destaque das cifras de áreas queimadas do Cerrado em relação aos demais biomas era esperado. Porém, isso não significa entender que não se deva atentar a que estas áreas queimem ou deixem de queimar, ou em que frequências e padrões sazonais ocorrem. É importante ressaltar que estes tipos de ecossistemas não são adaptados ao fogo, mas sim a determinados regimes de queima (PAUSAS; KEELEY, 2009). O Cerrado passou por constantes pressões antrópicas nas últimas décadas, sendo seus remanescentes de vegetação natural de $\approx 41 \%$ (SOARES-FILHO et al., 2014), o que resulta, direta e indiretamente, em alterações significativas dos seus regimes naturais de queima.

Em termos espaciais, é possível verificar uma assimetria na distribuição espacial de áreas queimadas no bioma Cerrado. Extensões mais contínuas e com alta frequência de fogo são observadas, principalmente, no seu setor meio-norte e norte, e nas transições com os biomas Amazônia e Pantanal, contrastando com outros setores onde o acúmulo de áreas queimadas é mais fragmentado e menos frequente (setores a sudoeste e sudeste, principalmente). Tratam-se de regiões que se distinguem muito em relação a densidade demográfica e principalmente ao uso e cobertura da terra, sendo as áreas mais afetadas onde se concentram maiores extensões de formação vegetal natural do Cerrado. Em contrapartida, os setores sudoeste e sudeste do Cerrado já apresentam alta densidade de fragmentação da paisagem natural, predominância de cobertura de pastagens e áreas agrícolas, que fragmentam a paisagem e podem atuar como barreiras para reduzir a propagação de grandes incêndios (ANDELA; VAN DER WERF, 2014).

Cabe pontuar que, ainda que os subtotais de áreas queimadas registrados nestes biomas sejam principalmente sobre formações naturais, não é possível afirmar que se tratam de queimas de origem natural. Mesmo que os raios sejam uma importante fonte de ignições de incêndios em ambientes savânicos (RAMOS-NETO; PIVELLO, 2000), muitas vezes desconsiderada, as ignições antrópicas ampliaram notavelmente nas últimas décadas, com o aumento 
significativo da ocupação de áreas de agropastoris que aliam o uso do fogo nas suas atividades. Queimadas com ignições antrópicas, que, intencionalmente ou não, avançam sobre áreas de cobertura vegetal natural (CANO-CRESPO et al., 2015), são uma importante fonte de ignições de incêndios.

Soma-se a isso que muitas áreas naturais remanescentes, situadas em Unidades de Conservação, passaram, dentro dos anos analisados, por um período em que uma política de fogo-zero foi aplicada, favorecendo o acúmulo de material combustível e a ocorrência de grandes incêndios (DURIGAN; RATTER, 2016; FIDELIS et al., 2018). Este panorama tem sido alterado nos últimos anos, com a adoção de políticas que contemplam de uma maneira mais adequada o papel do fogo de acordo com as características dos ecossistemas afetados (BERLINCK; BATISTA, 2020), fomentando o planejamento e execução de planos de manejo integrado do fogo no âmbito das políticas de conservação brasileiras (SCHMIDT et al., 2018).

Esta relação entre acúmulo de material combustível e favorecimento de grandes incêndios pode ser um dos fatores que auxiliam a explicar um dos padrões observados na variabilidade interanual de queimadas, onde certos anos de picos da série são precedidos de anos com poucas áreas queimadas. Esta situação é também retratada em pesquisas que levantam o histórico de fogo anual de áreas queimadas a escala regional, tais como observado no Enclave de Cerrado dos Campos Amazônicos (ALVES; PÉREZ-CABELLO, 2017; ALVES et al., 2018), no Parque Nacional da Serra do Cipó (ALVARADO et al., 2017) e no Parque Nacional da Serra das Confusões (ARGIBAY; SPARACINO; ESPINDOLA, 2020).

Porém, para além dos fatores associados ao acúmulo de material combustível, está a influência das anomalias climáticas regionais, e em especial da influência do El Niño Oscilação Sul (ENOS) e da Oscilação Multidecadal do Atlântico (OMA), que estão muitas vezes associadas a secas mais severas e intensas que favorecem a ocorrência de incêndios. $\mathrm{O}$ ano de 2010, por exemplo, ressaltado nos subtotais anuais de áreas queimadas como um pico na Amazônia, Cerrado, Caatinga e Pantanal, é um ano de forte seca (MARENGO et al., 2011). Em termos espaciais, a influência destas anomalias se manifesta de maneira heterogênea; ENOS está mais associado a queimadas no oeste da Amazônia, 
enquanto que as variações do OMA influenciam mais as queimadas no sul e sudoeste da Amazônia (CHEN et al., 2011).

Todos estes fatores ressaltam a importância de avaliar de maneira ampla as complexidades dos padrões de área queimada nos biomas brasileiros, para que se disponha de informações que recolham suas especificidades regionais.

\section{CONCLUSÕES E CONSIDERAÇÕES FINAIS}

As análises realizadas permitiram dar resposta aos objetivos inicialmente sinalizados, caracterizando-se genericamente os padrões de incidência de fogo nos diferentes biomas brasileiros nas últimas duas décadas em relação aos tipos de uso e cobertura da terra afetados. Comprova-se o potencial de combinação de uso de produtos derivados de sensoriamento remoto, e no caso concreto do produto MCD64A1 de áreas queimadas e dos dados de uso e cobertura da terra do Mapbiomas, para explorar a variação dos padrões espaço-temporais de ocorrência do fogo no território brasileiro.

Foi possível ressaltar a forte influência do fogo na dinâmica dos ecossistemas brasileiros, e, em especial, sobre aqueles que possuem uma relação evolutiva com fogo, como é o caso dos biomas Cerrado e Pantanal. Nestes ecossistemas, assim como na Caatinga, predominaram no período analisado as queimadas sobre áreas de vegetação natural (formações savânicas e campestres), enquanto que sobre os biomas Amazônia, Mata Atlântica e Pampa predominaram as áreas queimadas sobre setores agropastoris, com proporções superiores $\mathrm{a} \approx 50 \%$ dos subtotais afetados pelo fogo.

Compreender os atuais padrões espaço temporais de incidência do fogo exige dispor de informações que permitam analisar as influências antrópicas na formação e transformação das paisagens, abordando, portanto, aspectos ecológicos, culturais e socioeconômicos. É preciso entender os impactos das alterações dos regimes de queima sobre componentes da bioesfera, pedoesfera e atmosfera, mas é também necessário considerar a importância do fogo na dinâmica de ecossistemas que com este evoluíram, ampliando debates sobre o seu emprego para atividades agropastoris, valorizando e reconhecendo os saberes tradicionais associados ao seu uso, e fomentando e regularizando políticas de manejo integrado do fogo em áreas protegidas. A Geografia, engajada na 
compreensão das dinâmicas espaciais de inter-relação natureza-sociedade, tem muito a contribuir neste debate.

\section{AGRADECIMENTOS}

Os autores agradecem a FAPESP (processo n. 2019/o7357-8, vinculado ao processo JP 2015/06743-0), ao CNPq (processos n. 15466o/2018-3; e 441968/2018-o), e a UEMA (bolsa de fixação de doutor - res. 840/2018) pelo financiamento das atividades de pesquisa dos autores.

\section{REFERÊNCIAS}

ADENEY, J. M.; CHRISTENSEN, N. L.; PIMM, S. L. Reserves protect against deforestation fires in the Amazon. PloS one, v. 4, n. 4, p. e5014, 2009.

ALCAÑIZ, M.; OUTEIRO, L.; FRANCOS, M.; FARGUELL, J.; ÚBEDA, X. Longterm dynamics of soil chemical properties after a prescribed fire in a Mediterranean forest (Montgrí Massif, Catalonia, Spain). Science of the Total Environment, v. 572, p. 1329-1335, 2016.

ALENCAR, A.; ASNER, G. P.; KNAPP, D.; ZARIN, D. Temporal variability of forest fires in eastern Amazonia. Ecological Applications, v. 21, n. 7, p. 2397-412, 2011.

ALVARADO, S. T.; FORNAZARI, T.; CÓSTOLA, A.; MORELLATO, L. P. C.; SILVA, T. S. F. Drivers of fire occurrence in a mountainous Brazilian cerrado savanna: Tracking long-term fire regimes using remote sensing. Ecological Indicators, v. 78, p. 270-281, 2017.

ALVARADO, S. T.; SILVA, T. S. F.; ARCHIBALD, S. Management impacts on fire occurrence: A comparison of fire regimes of African and South American tropical savannas in different protected areas. Journal of Environmental Management, v. 218, p. 79-87, 2018.

ALVES, D. B.; PÉREZ-CABELLO, F. Multiple remote sensing data sources to assess spatio-temporal patterns of fire incidence over Campos Amazônicos Savanna Vegetation Enclave (Brazilian Amazon). Science of The Total Environment, v. 601-602, p. 142-158, 2017.

ALVES, D. B.; PÉREZ-CABELLO, F.; RODRIGUES MIMBRERO, M.; FEBRERMARTÍNEZ, M. Accuracy assessment of the latest generations of MODIS burned area products for mapping fire scars on a regional scale over Campos Amazônicos Savanna Enclave (Brazilian Amazon). Journal of Applied Remote Sensing, v. 12, n. 2, p. 1-21, 2018. 
ANDELA, N.; VAN DER WERF, G. R. Recent trends in African fires driven by cropland expansion and El Niño to La Niña transition. Nature Climate Change, v. 4, n. 9, p. 791-795, 2014.

ARAGÃO, L. E. O. C.; MALHI, Y.; BARBIER, N.; LIMA, A.; SHIMABUKURO, Y. E.; ANDERSON, L.; SAATCHI, S. Interactions between rainfall, deforestation and fires during recent years in the Brazilian Amazonia. Philosophical transactions of the Royal Society of London B: Biological sciences, v. 363, n. 1498, p. 1779-85, 2008.

ARCHER, S. R.; ANDERSEN, E. M.; PREDICK, K. I.; SCHWINNING, S.; STEIDL, R. J.; WOODS, S. R. Woody Plant Encroachment: Causes and Consequences. In: BRISKE, D. D. (Ed.). Rangeland Systems: Processes, Management and Challenges. Springer Series on Environmental Management. Cham, Switzerland: Springer International Publishing, 2017. p. 25-84.

ARGIBAY, D. S.; SPARACINO, J.; ESPINDOLA, G. M. A long-term assessment of fi re regimes in a Brazilian ecotone between seasonally dry tropical forests and savannah. Ecological Indicators, v. 113, p. 1-13, 2020.

BALCH, J. K.; MASSAD, T. J.; BRANDO, P. M.; NEPSTAD, D. C.; CURRAN, L. M. Effects of high-frequency understorey fires on woody plant regeneration in southeastern Amazonian forests. Philosophical Transactions of the Royal Society B: Biological Sciences, v. 368, n. 1619, p. 20120157-20120157, 2013.

BATISTA, E. K. L.; RUSSELL-SMITH, J.; FRANÇA, H.; FIGUEIRA, J. E. C. An evaluation of contemporary savanna fire regimes in the Canastra National Park, Brazil: Outcomes of fire suppression policies. Journal of Environmental Management, v. 205, p. 40-49, 2018.

BERLINCK, C. N.; BATISTA, E. K. L. Good fire, bad fire: it depends on who burns. Flora, v. 268, n. December 2019, 2020.

BOND, W. J.; WOODWARD, F. I.; MIDGLEY, G. F. The Global Distribution of Ecosystems in a world without Fire. New Phytologist, v. 165, n. 2, p. 525-538, 2005 .

BOWMAN, D. M. J. S.; BALCH, J. K.; ARTAXO, P.; BOND, W. J.; CARLSON, J. M.; COCHRANE, M. A.; ANTONIO, C. M. D.; DEFRIES, R. S.; DOYLE, J. C.; HARRISON, S. P.; JOHNSTON, F. H.; KEELEY, J. E.; KRAWCHUK, M. A.; KULL, C. A.; MARSTON, J. B.; MORITZ, M. A.; PRENTICE, I. C.; ROOS, C. I.; SCOTT, A. C.; SWETNAM, T. W.; VAN DER WERF, G. R.; PYNE, S. J. Fire in the Earth System. Science, v. 324, n. 5926, p. 481-484, 2009.

BRANDO, P. M.; BALCH, J. K.; NEPSTAD, D. C.; MORTON, D. C.; PUTZ, F. E.; COE, M. T.; SILVÉRIO, D.; MACEDO, M. N.; DAVIDSON, E. A.; NÓBREGA, C. C.; ALENCAR, A.; SOARES-FILHO, B. S. Abrupt increases in Amazonian tree mortality due to drought-fire interactions. Proceedings of the National Academy of Sciences of the United States of America, v. 111, n. 17, p. 6347-52, 2014. 
CANO-CRESPO, A.; OLIVEIRA, P. J. C.; BOIT, A.; CARDOSO, M.; THONICKE, $\mathrm{K}$. Forest edge burning in the Brazilian Amazon promoted by escaping fires from managed pastures. Journal of Geophysical Research: Biogeosciences, v. 120, n. 10, p. 2095-2107, 2015.

CARDOZO, S.; PEREIRA, G.; SHIMABUKURO, Y. E.; MORAES, E. C. Analysis and assessment of the spatial and temporal distribution of burned areas in the Amazon Forest. Remote Sensing, n. 6, p. 8002-8025, 2014.

CERTINI, G. Effects of fire on properties of forest soils: A review. Oecologia, v. 143, n. 1, p. 1-10, 2005.

CHEN, Y.; RANDERSON, J. T.; MORTON, D. C.; DEFRIES, R. S.; COLLATZ, G. J.; KASIBHATLA, P. S.; GIGLIO, L.; JIN, Y.; MARLIER, M. E. Forecasting fire season severity in South America using sea surface temperature anomalies.

Science, v. 334, n. Nov., p. 787-792, 2011.

CRED, Centre for Research on the Epidemiology of Disasters. Natural Disasters 2017, Disponível em: <

https://www.preventionweb.net/publications/view/60351>. Acesso em 3 abril de 2020.

CRED, Centre for Research on the Epidemiology of Disasters. Natural Disasters 2018, Disponível em:

<https://www.preventionweb.net/publications/view/66133>. Acesso em 3 abril de 2020.

CRED, Centre for Research on the Epidemiology of Disasters. Disaster year in review, 2020. Disponível em: <

https://www.preventionweb.net/publications/view/71642>. Acesso em 3 abril de 2020.

DALDEGAN, G. A.; DE CARVALHO JÚNIOR, O. A.; GUIMARÃES, R. F.; GOMES, R. A. T.; RIBEIRO, F. de F.; MCMANUS, C. Spatial patterns of fire recurrence using remote sensing and GIS in the Brazilian savanna: Serra do Tombador Nature Reserve, Brazil. Remote Sensing, v. 6, n. 10, p. 9873-9894, 2014 .

DURIGAN, G.; RATTER, J. A. The need for a consistent fire policy for Cerrado conservation. Journal of Applied Ecology, v. 53, n. 1, p. 11-15, 2016.

EVA, H.; LAMBIN, E. F. Fires and land-cover change in the tropics: a remote sensing analysis at the landscape scale. Journal of Biogeography, v. 27, n. 3, p. 765-776, 2000.

FIDELIS, A.; ALVARADO, S.; BARRADAS, A.; PIVELLO, V. The Year 2017: Megafires and Management in the Cerrado. Fire, v. 1, n. 3, p. 49, 2018.

GIGLIO, L.; BOSCHETTI, L.; ROY, D.; HOFFMAN, A. A.; HUMBER, M. Collection MODIS 6 Burned Area Product User's Guide. Disponível em: 
<https://lpdaac.usgs.gov/sites/default/files/public/product_documentation/m cd64_user_guide_v6.pdf $>$. Acesso em 3 setembro de 2019.

GIGLIO, L.; BOSCHETTI, L.; ROY, D. P.; HUMBER, M. L.; JUSTICE, C. O. The Collection 6 MODIS burned area mapping algorithm and product. Remote Sensing of Environment, v. 217, n. July, p. 72-85, 2018.

GIGLIO, L.; RANDERSON, J. T.; VAN DER WERF, G. R.; KASIBHATLA, P. S.; COLLATZ, G. J.; MORTON, D. C.; DEFRIES, R. S. Assessing variability and long-term trends in burned area by merging multiple satellite fire products. Biogeosciences, v. 7, p. 1171-1186, 2010.

GOLDAMMER, J. G. Historical biogeography of fire: tropical and subtropical. In: CRUTZEN, P. J.; GOLDAMMER, J. G. (Ed.). The ecological, atmospheric and climatic importance of vegetation fires. New York: John Wiley \& Sons, Ltd, 1993. p. 297-314.

GORELICK, N.; HANCHER, M.; DIXON, M.; ILYUSHCHENKO, S.; THAU, D.; MOORE, R. Google Earth Engine: Planetary-scale geospatial analysis for everyone. Remote Sensing of Environment, v. 202, p. 18-27, 2017.

GREENVILLE, A. C.; DICKMAN, C. R.; WARDLE, G. M.; LETNIC, M. The fire history of an arid grassland: The influence of antecedent rainfall and ENSO. International Journal of Wildland Fire, v. 18, n. 6, p. 631-639, 2009.

HARDESTY, J.; MYERS, R.; FULKS, W. Fire, ecosystems and people: a preliminary assessment of fire as a global conservation issue. Fire Management, v. 22, n. 4, p. 78-87, 2005.

HE, T.; LAMONT, B. B.; PAUSAS, J. G. Fire as a key driver of Earth's biodiversity. Biological Reviews, v. 94, n. 6, p. 1983-2010, 2019.

HOFFMANN, W. A.; MOREIRA, A. G. The role of fire in population dynamics of woody plants. In: OLIVEIRA, P. S.; MARQUIS, R. . (Ed.). The Cerrados of Brazil: Ecology and Natural History of a Neotropical Savanna. New York, EUA: Columbia University Press, 2002. p. 139-177.

HUDAK, A. T.; BROCKETT, B. H. Mapping fire scars in a southern African savannah using Landsat imagery. International Journal of Remote Sensing, v. 25, n. 16, p. 3231-3243, ago. 2004.

HUMBER, M. L.; BOSCHETTI, L.; GIGLIO, L.; JUSTICE, C. O. Spatial and temporal intercomparison of four global burned area products. International Journal of Digital Earth, v. 8947, p. 1-25, 2018.

JACQUIN, A.; GOULARD, M. Using Spatial Statistics Tools on Remote-Sensing Data to Identify Fire Regime Linked with Savanna Vegetation Degradation.

International Journal of Agricultural and Environmental Information Systems, v. 4, n. 1, p. 68-82, 2013. 
LEMES, G. P.; MATRICARDI, E. A. T.; COSTA, O. B.; LEAL, F. A. Spatiotemporal assessment of forest fires occurred in the Serra da Canastra National Park between 1991 and 2011. Ambiência, v. 10, n. 1, p. 247-266, 2014 .

LEVIN, N.; HEIMOWITZ, A. Mapping spatial and temporal patterns of Mediterranean wildfires from MODIS. Remote Sensing of Environment, v. 126, p. 12-26, 2012.

LEVINE, J. S.; COFER, W. R.; CAHOON, D. J.; WINSTEAD, E. L. Biomass burning - a driver for global change. Environmental Science \& Technology, v. 29, n. 3, p. 120-125, 1995.

MAPBIOMAS. Projeto Mapbiomas - Coleção 4.1 da série anual de mapas de cobertura e uso de solo do Brasil. Disponível em: <http://mapbiomas.org/>. Acesso em 18 de abril 2020.

MARENGO, J. A.; TOMASELLA, J.; ALVES, L. M.; SOARES, W. R.; RODRIGUEZ, D. A. The drought of 2010 in the context of historical droughts in the Amazon region. Geophysical Research Letters, v. 38, n. 12, p. 1-5, 2011.

MOREIRA, A. G. Effects of fire protection on savanna structure in central Brazil. Journal of Biogeography, v. 27, n. 4, p. 1021-1029, 2000.

MORTON, D. C.; DEFRIES, R. S.; NAGOL, J.; SOUZA JR., C. M.; KASISCHKE, E. S.; HURTT, G. C.; DUBAYAH, R. Mapping canopy damage from understory fires in Amazon forests using annual time series of Landsat and MODIS data. Remote Sensing of Environment, v. 115, n. 7, p. 1706-1720, 2011.

MOUILLOT, F.; SCHULTZ, M. G.; YUE, C.; CADULE, P.; TANSEY, K.; CIAIS, P.; CHUVIECO, E. Ten years of global burned area products from spaceborne remote sensing-A review: Analysis of user needs and recommendations for future developments. International Journal of Applied Earth Observation and Geoinformation, v. 26, n. 1, p. 64-79, 2014.

NEPSTAD, D.; CARVALHO, G.; BARROS, A. C.; ALENCAR, A.; CAPOBIANCO, J. P.; BISHOP, J.; MOUTINHO, P.; LEFEBVRE, P.; SILVA JR., U. L.; PRINS, E. Road paving, fire regime feedbacks, and the future of Amazon forests. Forest Ecology and Management, v. 154, p. 397-407, 2001.

OMETTO, J. P.; SOUZA-NETO, E. r.; TEJADA, G. Land Use, Land Cover and Land Use Change in the Brazilian Amazon (1960-2013). In: NAGY, L.; FORSBERG, B. R.; ARTAXO, P. (Ed.). Interactions between biosphere, atmosphere and human land use Amazon Basin. Ecological Studies. Berlin, Heidelberg: Springer Berlin Heidelberg, 2016. 227p. 369-384.

PADILLA, M.; STEHMAN, S. V.; RAMO, R.; CORTI, D.; HANTSON, S.; OLIVA, P.; ALONSO-CANAS, I.; BRADLEY, A. V.; TANSEY, K.; MOTA, B.; PEREIRA, J. M.; CHUVIECO, E. Comparing the accuracies of remote sensing global burned 
area products using stratified random sampling and estimation. Remote Sensing of Environment, v. 160, n. APRIL, p. 114-121, 2015.

PAUSAS, J. G.; KEELEY, J. E. A burning story: The role of fire in the history of life. BioScience, v. 59, n. 7, p. 593-601, 2009.

PÉREZ-CABELLO, F.; ECHEVERRÍA, M. T.; IBARRA, P.; DE LA RIVA, J. Effects of Fire on Vegetation, Soil and Hydrogeomorphological Behavior in Mediterranean Ecosystems. In: CHUVIECO, E. (Ed.). Earth Observation of Wildland Fires in Mediterranean Ecosystems. Berlin, Heidelberg: Springer Berlin Heidelberg, 2009. p. 111-128.

PIVELLO, V. R. The use of fire in the cerrado and amazonian rainforests of Brazil: past and present. Fire Ecology, v. 7, n. 1, p. 24-39, abr. 2011.

RAMOS-NETO, M. B.; PIVELLO, V. R. Lightning fires in a Brazilian Savanna National Park: rethinking management strategies. Environmental Management, v. 26, n. 6, p. 675-684, 2000.

RCT, R Core Team. R: A Language and Environment for Statistical Computing Vienna, Austria: R Foundation for Statistical Computing, 2019.

RÖDER, A.; BÄRISCH, S.; HILL, J.; DUGUY, B.; ALLOZA, J. A.; VALLEJO, R. An interpretation framework for fire events and post-fire dynamics in Ayora / Spain using time-series of Landsat-TM and -MSS data. New Strategies for European Remote Sensing, p. 51-60, 2005.

ROY, D. P.; LEWIS, P. E.; JUSTICE, C. O. Burned area mapping using multitemporal moderate spatial resolution data-a bi-directional reflectance modelbased expectation approach. Remote Sensing of Environment, v. 83, n. 12, p. 263-286, 2002.

SCHMIDT, I. B.; MOURA, L. C.; FERREIRA, M. C.; ELOY, L.; SAMPAIO, A. B.; DIAS, P. A.; BERLINCK, C. N. Fire management in the Brazilian savanna: First steps and the way forward. Journal of Applied Ecology, n. October 2017, p. 1-8, 2018.

SCHROEDER, W.; ALENCAR, A.; ARIMA, E.; SETZER, A. The spatial distribution and interannual variability of fire in Amazonia. In: KELLER, M.; BUSTAMANTE, M.; GASH, J.; DIAS, P. S. (Ed.). Amazonia and Global Change. Washington, DC, United States: American Geophysical Union, 2009. p. 43-60.

SCOTT, A. C.; GLASSPOOL, I. J. The diversification of Paleozoic fire systems and fluctuations in atmospheric oxygen concentration. Proceedings of the National Academy of Sciences of the United States of America, v. 103, n. 29, p. 10861-10865, 2006. 
SILVA CARDOZO, F. da; SHIMABUKURO, Y. E.; PEREIRA, G.; SILVA, F. B. Using remote sensing products for environmental analysis in South America. Remote Sensing, v. 3, n. 10, p. 2110-2127, 2011.

SIMON, M. F.; GRETHER, R.; DE QUEIROZ, L. P.; SKEMA, C.; PENNINGTON, R. T.; HUGHES, C. E. Recent assembly of the Cerrado, a neotropical plant diversity hotspot, by in situ evolution of adaptations to fire. Proceedings of the National Academy of Sciences, v. 106, n. 48, p. 20359-20364, 1 dez. 2009.

SOARES-FILHO, B.; RAJÃO, R.; MACEDO, M.; CARNEIRO, A.; COSTA, W.; COE, M.; RODRIGUES, H.; ALENCAR, A. Cracking Brazil's Forest Code.

Science, v. 344, n. 6182, p. 363-364, 2014.

STEVENS, N.; LEHMANN, C. E. R.; MURPHY, B. P.; DURIGAN, G. Savanna woody encroachment is widespread across three continents. Global Change Biology, v. 23, n. 1, p. 235-244, 2017.

TSELA, P.; WESSELS, K.; BOTAI, J.; ARCHIBALD, S.; SWANEPOEL, D.; STEENKAMP, K.; FROST, P. Validation of the two standard MODIS satellite burned-area products and an empirically-derived merged product in South Africa. Remote Sensing, v. 6, n. 2, p. 1275-1293, 2014.

VAN DER WERF, G. R.; RANDERSON, J. T.; GIGLIO, L.; COLLATZ, G. J.; MU, M.; KASIBHATLA, P. S.; MORTON, D. C.; DEFRIES, R. S.; JIN, Y.; VAN LEEUWEN, T. T. Global fire emissions and the contribution of deforestation, savanna, forest, agricultural, and peat fires (1997-2009). Atmospheric Chemistry and Physics, v. 10, n. 23, p. 11707-11735, 2010.

VAN DER WERF, G. R.; RANDERSON, J. T.; GIGLIO, L.; VAN LEEUWEN, T. T.; CHEN, Y.; ROGERS, B. M.; MU, M.; VAN MARLE, M. J. E.; MORTON, D. C.; COLLATZ, G. J.; YOKELSON, R. J.; KASIBHATLA, P. S. Global fire emissions estimates during 1997-2015. Earth System Science Data Discussions, n. January, p. 1-43, 2017. 